\title{
Experimental comparison between nonholonomic and vakonomic mechanics in nonlinear constrained systems
}

\author{
Tatsuya $\mathrm{Kai}^{1 \mathrm{a})}$ \\ ${ }^{1}$ Faculty of Industrial Science and Technology, Tokyo University of Science, \\ 6-3-1 Niijuku, Katsushika-ku, Tokyo 125-8585, Japan \\ a)kai@rs.tus.ac.jp
}

Received January 25, 2013; Revised June 9, 2013; Published October 1, 2013

\begin{abstract}
It is known that there exist two types of methods to derive the equations of motion for mechanical systems subject to constraints. They are called nonholonomic and vakonomic mechanics. Lewis and Murray showed some simulations for two models of a ball on a rotating table, which are modeled by nonholonomic and vakonomic mechanics, and carried out experiments using a laboratory equipment. They concluded that nonholonomic mechanics provides a good model for a ball on a rotating table. However, they did not show the details of experiments, and hence there exist some ambiguous parts in their paper. Hence, we perform additional experiments for a ball on a rotating table using a laboratory equipment of a ball on a rotating table, and compare simulation and experimental results. In this manner, we can confirm that nonholonomic mechanics shows better performances in comparison with vakonomic mechanics as Lewis and Murray asserted.
\end{abstract}

Key Words: nonlinear systems, constrained systems, nonholonomic mechanics, vakonomic mechanics, a ball on a rotating table

\section{Introduction}

In the research fields such as control theory and robotics, studies on nonholonomic systems have been actively done so far [1-4]. Simply stated, nonholonomic systems are defined as systems whose motions are subject to nonintegrable constraints [5]. One can find a lot of examples of nonholonomic systems in physics and mechanical engineering: mobile cars, trailers, hopping robots, underactuated manipulators, space robots, a ball/coin on a plane, a ball on a rotating table and so on. Researches on nonholonomic systems originally started in the field of analytical mechanics, and it is said that the motivation is the fact that the equation of motion for the system of a rolling coin on a plane (called the rolling penny) cannot be derived by Euler-Lagrange equations.

From the 19th century, various researchers such as Caplygin, Synge, Pars, Hölder, Chetaev, Capon, Hertz and Lindelöf tackled the problem of how to realize constraints in the equations of motion [5]. As a result of these studies, nonholonomic mechanics based on d'Alembert's principle has become widely used for derivation of the equations of motion for systems subject to constraints. Meanwhile, Ko- 
zlov proposed vakonomic mechanics (mechanics of variational axiomatic kind) which is a completely different method from nonholonomic mechanics and based on Hamilton's principle of least action in 1983 [7]. The equations of motion derived by vakonomic mechanics are fundamentally different in those obtained by nonholonomic mechanics, and this fact caused a paradoxical situation that there exist two different methods to derive the equations of motion for a system. For the problem of which mechanics shows realistic behaviors, Lewis and Murray compared between these two mechanics from the viewpoints of simulations and experiments in 1995 [9]. The authors dealt with a ball on a rotating table as an example of a nonholonomic system, and performed simulations and experiments for the system. The authors concluded that the equation of motion derived by nonholonomic mechanics gives a good model for the system of a ball on a rotating table. However, the details of the experiments such as illustrations of trajectories of the ball and pictures of the experiments were not presented in the paper, and hence there remain some ambiguous parts in the paper.

The purpose of this paper is that we carry out additional tests of experiments for the system of a ball on a rotating table and compare experimental results with simulation ones in order to confirm Lewis and Murray's assertion. The outline of this paper is as follows. In section 2, we give a summary on nonholonomic and vakonomic mechanics. Section 3 presents the problem setting of the system of a ball on a rotating table, and these two models are derived via nonholonomic and vakonomic mechanics, which are called the nonholonomic and vakonomic equations. In Section 4, we perform some simulations of both the nonholonomic and vakonomic equations, and give theoretical analysis on the relationship between the both models. In Section 5, we carry out some laboratory experiments of a ball on a rotating table, and we compare the experimental results with simulations obtained in Section 4 .

\section{Nonholonomic and vakonomic mechanics}

In this section we give a summary on some important concepts of modeling methods for mechanical systems subject to constraints. Especially, we focus on nonholonomic and vakonomic mechanics. We base our exposition in this section on the work of Lewis and Murray [9], to which we refer for further information. For more details of nonholonomic and vakonomic mechanics, see the references $[6,8,10-$ $18]$.

First, we consider the case where there exists no constraint in a system as preliminaries. Let $Q$ be an $n$-dimensional smooth configuration manifold with a generalized coordinates $q=\left[q_{1} \cdots q_{n}\right]^{\top} \in \mathbf{R}^{n}$, and $\dot{q}=\left[\dot{q}_{1} \cdots \dot{q}_{n}\right]^{\top} \in \mathbf{R}^{n}$ be a generalized velocity that belongs to the tangent space $T_{q} Q$. Hence, the coordinate of the tangent bundle $T Q$ is represented by the pair $(q, \dot{q})$. Let us denote a time interval by $I=\left[t_{0}, t_{1}\right]$. For given two points $q_{0}, q_{1} \in Q$, we define the manifold of twice differentiable curves $q(t)$ which are connected from $q_{0}$ to $q_{1}$ as

$$
C\left(q_{0}, q_{1}, I\right)=\left\{q: I \rightarrow Q \mid q \in \mathcal{C}^{2} ; q\left(t_{0}\right)=q_{0}, q\left(t_{1}\right)=q_{1}\right\} .
$$

In addition, we consider a Lagrangian function $L: T Q \rightarrow \boldsymbol{R}$ and we define a functional $F$ : $C\left(q_{0}, q_{1}, I\right) \rightarrow \boldsymbol{R}$ for a curve $q(t) \in C\left(q_{0}, q_{1}, I\right)$ as

$$
F(q)=\int_{t_{0}}^{t_{1}} L(q(t), \dot{q}(t)) d t
$$

Then, the tangent space of $C\left(q_{0}, q_{1}, I\right)$ at a point $q$ is given by

$$
T_{q} C\left(q_{0}, q_{1}, I\right)=\left\{X: I \rightarrow T Q \mid q \in \mathcal{C}^{1} ; X(t) \in T_{q(t)} Q, \forall t \in I ; X\left(t_{0}\right)=X\left(t_{1}\right)=0\right\},
$$

where $X$ is called a variation. Now, we deal with the following variational problem.

Problem 1 [Variational Problem] Define an increment of the functional $F(q)$ by a variation $X$ as $\delta F(q)(X)$. Find a curve $q^{*}(t) \in C\left(x_{0}, x_{1}, I\right)$ such that $F(q)$ is stationary for all variations $X \in T_{q} C\left(q_{0}, q_{1}, I\right)$, that is, $\delta F\left(q^{*}\right)(X)=0, \forall X \in T_{q} C\left(q_{0}, q_{1}, I\right)$ holds. 
As a solution of the variational problem above, we can derive the following well-known result.

Theorem 1 [Euler-Lagrange Equations] : A curve $q^{*}(t) \in C\left(q_{0}, q_{1}, I\right)$ is a solution of the variational problem (Problem 1 ), that is, $q^{*} \in C\left(q_{0}, q_{1}, I\right)$ is a stationary point of the functional $F(q)$ for all variations $X \in T_{q} C\left(q_{0}, q_{1}, I\right)$ if and only if $q^{*}(t)$ satisfies

$$
\frac{d}{d t}\left(\frac{\partial L}{\partial \dot{q}}\right)-\frac{\partial L}{\partial q}=0 .
$$

(4) is called Euler-Lagrange equations.

(Proof) Using integration by parts, we can calculate the increment of the functional $\delta F(q)(X)$ for a variation $X$ as follows.

$$
\begin{aligned}
\delta F(q)(X) & =\int_{t_{0}}^{t_{1}}\{L(q+X, \dot{q}+\dot{X})-L(q, \dot{q})\} d t \\
& =\int_{t_{0}}^{t_{1}}\left(\left\langle\frac{\partial L}{\partial q}, X\right\rangle+\left\langle\frac{\partial L}{\partial \dot{q}}, \dot{X}\right\rangle\right) d t \\
& =\int_{t_{0}}^{t_{1}}\left\{\left\langle\frac{\partial L}{\partial q}, X\right\rangle+\frac{d}{d t}\left\langle\frac{\partial L}{\partial \dot{q}}, X\right\rangle-\left\langle\frac{d}{d t}\left(\frac{\partial L}{\partial \dot{q}}\right), X\right\rangle\right\} d t \\
& =\left[\left\langle\frac{\partial L}{\partial \dot{q}}, X\right\rangle\right]_{t_{0}}^{t_{1}}+\int_{t_{0}}^{t_{1}}\left\langle\frac{\partial L}{\partial q}-\frac{d}{d t}\left(\frac{\partial L}{\partial \dot{q}}\right), X\right\rangle d t,
\end{aligned}
$$

where $\langle\cdot, \cdot\rangle$ is the inner product. Using $X\left(t_{0}\right)=X\left(t_{1}\right)=0$, we then have

$$
\delta F(q)(X)=\int_{t_{0}}^{t_{1}}\left\langle\frac{\partial L}{\partial q}-\frac{d}{d t}\left(\frac{\partial L}{\partial \dot{q}}\right), X\right\rangle d t .
$$

Since $\delta F(q)(X)=0$ must be satisfied for all variation $X$, we obtain (4).

Next, the case where there exist constraints in a system is discussed. If a system subject to some constraints, we have to take them into account in modeling of the system. However, there are two different methods to derive the equations of motion for the constrained system. One is called nonholonomic mechanics which is well known since early times. The other is called vakonomic mechanics and originally introduced by Kozlov in 1983 [7]. We will summarize the derivation methods and the characteristics of these two mechanics. We now assume that the system is subject to $m$ constraints in the form:

$$
\Phi(q, \dot{q})=\left[\begin{array}{c}
\phi_{1}(q, \dot{q}) \\
\vdots \\
\phi_{m}(q, \dot{q})
\end{array}\right]=0 .
$$

We can see that the constraints (6) determine a subbundle $P$ of the tangent bundle $T Q$. So, we define a new submanifold of $C\left(q_{0}, q_{1}, I\right)$ using $P$ as

$$
\tilde{C}\left(q_{0}, q_{1}, I, P\right)=\left\{\tilde{q} \in C\left(q_{0}, q_{1}, I\right) \mid \dot{\tilde{q}}(t) \in P, \forall t \in I\right\} .
$$

A variation which satisfies the constraints (6) is called a constrained variation. In derivations of the equations of motion via nonholonomic and vakonomic mechanics, the constrained variation plays an important role.

Nonholonomic mechanics is a classical variational method to derive the equations of motion for systems subject to constraints. This method is basically based on d'Alembert's Principles, which states that "a virtual work which a constraint force does is equal to zero." That is to say, we can apply constraints to the system by adding a constraint force. In nonholonomic mechanics, constraints 
are applied after making the functional stationary. Consider the tangent space of $C\left(q_{0}, q_{1}, I\right)$ at $\tilde{q} \in \tilde{C}\left(q_{0}, q_{1}, I, P\right)$ :

$$
T_{\tilde{q}} C\left(q_{0}, q_{1}, I\right)=\left\{X: I \rightarrow T Q \mid X \in \mathcal{C}^{1} ; X(t) \in T_{\tilde{q}(t)} Q, \forall t \in I ; X\left(t_{0}\right)=X\left(t_{1}\right)=0\right\},
$$

and define the set of variations which belong to $P$ as

$$
\mathcal{X}_{\tilde{q}}=\left\{X \in T_{\tilde{q}} C\left(q_{0}, q_{1}, I\right) \mid\left(\frac{\partial \Phi}{\partial \dot{q}}\right)^{\top} X=0\right\} .
$$

Now, we deal with the following problem called the nonholonomic constrained variational problem.

Problem 2 [Nonholonomic Constrained Variational Problem] Find a curve $\tilde{q}^{*} \in$ $\tilde{C}\left(q_{0}, q_{1}, I, P\right)$ such that $F(q)$ is stationary for all variations $X \in \mathcal{X}_{\tilde{q}}$, that is, $\delta F\left(\tilde{q}^{*}\right)(X)=$ $0, \forall X \in \mathcal{X}_{\tilde{q}}$ holds.

A solution of the nonholonomic variational problem can be obtained by using d'Alembert's Principles as follows.

Theorem 2 [Nonholonomic Equations] : A curve $\tilde{q}^{*}(t) \in \tilde{C}\left(q_{0}, q_{1}, I, P\right)$ is a solution of the nonholonomic constrained variational problem (Problem 2), that is, $\tilde{q}^{*} \in \tilde{C}\left(q_{0}, q_{1}, I, P\right)$ is a stationary point of the functional $F(q)$ for all variations $X \in \mathcal{X}_{\tilde{q}}$ if and only if there exists a Lagrange multiplier $\mu \in \mathbf{R}^{m}$ such that

$$
\frac{d}{d t}\left(\frac{\partial L}{\partial \dot{q}}\right)-\frac{\partial L}{\partial q}=\left(\frac{\partial \Phi}{\partial \dot{q}}\right)^{\top} \mu
$$

holds for $\tilde{q}^{*}(t)$. (10) is called the nonholonomic equation.

(Proof) Consider the gradient vector of $\phi_{i}$ :

$$
\nabla \phi_{i}=\left[\begin{array}{c}
\frac{\partial \phi_{i}}{\partial \dot{q}_{1}} \\
\vdots \\
\frac{\partial \phi_{i}}{\partial \dot{q}_{n}}
\end{array}\right], i=1, \cdots, m .
$$

Since all the $m$ gradient vectors $\nabla \phi_{i}(i=1, \cdots, m)$ are orthogonal to $P=\Phi^{-1}(0)$, we can write the constraints force $f_{q}$ as

$$
f_{q}=\left(\frac{\partial \Phi}{\partial \dot{q}}\right)^{\top} \mu
$$

where $\mu$ is a Lagrange multiplier vector. From d'Alembert's principle, we can add this force to the right side of Euler-Lagrange equations (4). Therefore, we obtain (10).

Figure 1 [a] illustrates an image on derivation of the equations of motion in nonholonomic mechanics. It is noted that in nonholonomic mechanics the constrained space is not considered at first, however, the obtained curve is projected to the constrained space using the Lagrange multiplier and the constraint force. In the nonholonomic equation (10), there exists the Lagrange multiplier $\mu$ in the right-hand side of it, however, we can eliminate it using the equations of the constraints (6).

On the other hand, vakonomic mechanics is fundamentally based on Hamilton's principle of least action, which states "only a motion that makes the functional stationary is realized." Contrary to 


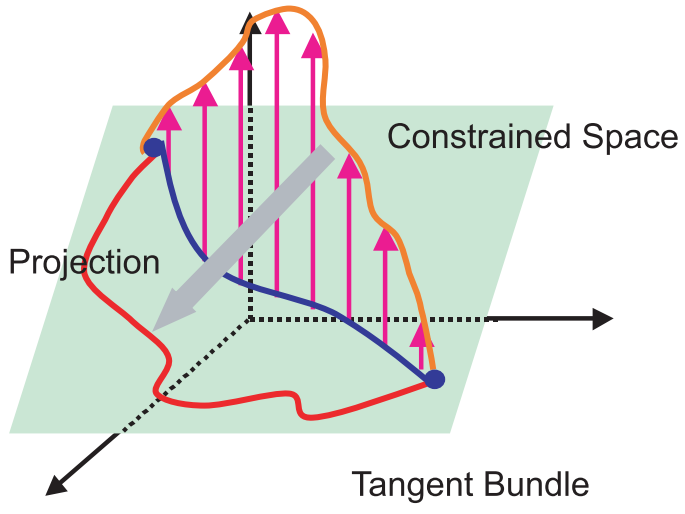

[a] Nonholonomic Mechanics

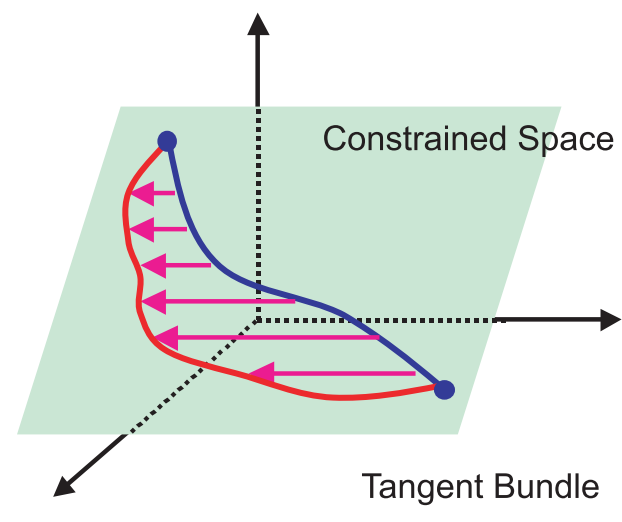

[b] Vakonomic Mechanics

Fig. 1. Variations in nonholonomic and vakonomic mechanics.

nonholonomic mechanics, in vakonomic mechanics constraints are applied before making the functional stationary. The tangent space of $\tilde{C}\left(q_{0}, q_{1}, I, P\right)$ at $\tilde{q} \in \tilde{C}\left(x_{0}, x_{1}, I, P\right)$ is given by

$$
\begin{aligned}
T_{\tilde{q}} \tilde{C}\left(q_{0}, q_{1}, I, P\right)=\left\{X: I \rightarrow T Q \mid X \in \mathcal{C}^{1}\right. & \\
& \left.X(t) \in P, \forall t ; X\left(t_{0}\right)=X\left(t_{1}\right)=0\right\} .
\end{aligned}
$$

We then consider the next problem called the vakonomic constrained variational problem.

Problem 3 [Vakonomic Constrained Variational Problem] Find a curve $\tilde{q}^{*} \in$ $\tilde{C}\left(q_{0}, q_{1}, I, P\right)$ such that $F(q)$ is stationary for all constrained variations $\tilde{X} \in T_{\tilde{q}} \tilde{C}\left(q_{0}, q_{1}, I, P\right)$, that is, $\delta F\left(\tilde{q}^{*}\right)(\tilde{X})=0, \forall \tilde{X} \in T_{\tilde{q}} \tilde{C}\left(q_{0}, q_{1}, I, P\right)$ holds.

In order to solve the vakonomic constrained variational problem, we show two useful theorems. Let $M$ and $N$ be smooth manifolds, and consider a mapping $g: M \rightarrow N$. If both $g$ and its derivative $\dot{g}: T_{p} M \rightarrow T_{g(p)} N$ are surjections for all $p \in M, g$ is called a submersion. Now, the submersion theorem is shown as follows $[6,9]$.

Theorem 3 [Submersion Theorem] : Let $M$ and $N$ be smooth manifolds. If a mapping $g: M \rightarrow N$ is a submersion, then $P=g^{-1}(0)$ is a smooth submanifold of $M$ and the dimension of $P$ is equal to $\operatorname{dim} M-\operatorname{dim} N$.

In addition, another important theorem called the Lagrange multiplier theorem is shown as follows $[6,9]$.

Theorem 4 [Lagrange Multiplier Theorem] : Let $M$ and $N$ be smooth manifolds. Consider a smooth submersion $g: M \rightarrow N$ and a mapping $f: M \rightarrow \boldsymbol{R}$. Then, $p$ is critical point of $f \mid P$ if and only if there exists a mapping $\lambda: N \rightarrow \boldsymbol{R}$ such that $p \in P$ is a stationary point of $f-\lambda \circ g$.

Using the submersion theorem and the Lagrange multiplier theorem, we can solve the vakonomic constrained variational problem as follows. 
Theorem 5 [Vakonomic Equations] : A curve $\tilde{q}^{*}(t) \in \tilde{C}\left(q_{0}, q_{1}, I, P\right)$ is a solution of vakonomic constrained variational problem (Problem 3), that is, $\tilde{q}^{*} \in \tilde{C}\left(q_{0}, q_{1}, I, P\right)$ is a stationary point of the functional $F(c)$ for all constrained variations $\tilde{X} \in T_{\tilde{q}} \tilde{C}\left(q_{0}, q_{1}, I, P\right)$ if there exists a Lagrange multiplier $\lambda \in \mathbf{R}^{m}$ such that

$$
\frac{d}{d t}\left(\frac{\partial \hat{L}}{\partial \dot{q}}\right)-\frac{\partial \hat{L}}{\partial q}=0
$$

holds for $\tilde{q}^{*}(t)$. where $\hat{L}$ is called an extended Lagrangian defined by

$$
\hat{L}(q, \dot{q})=L(q, \dot{q})-\lambda^{T} \Phi(q, \dot{q}) .
$$

(14) is called the vakonomic equation.

(Proof) In the Lagrange multiplier theorem, we identify the manifold $M$ with a $2 n$-dimensional tangent bundle $T Q$, the mapping $f$ with the constraints $\Phi(q, \dot{q}): T Q \rightarrow N$ and the mapping $g$ with the Lagrangian $L(q, \dot{q}): T Q \rightarrow \boldsymbol{R}$, respectively. Then, we can identify the manifold $N$ with an $m$-dimensional constrained space. From the submersion theorem, $P$ is a $(2 n-m)$-dimensional subbundle of $T Q$. Now, we consider a new function called the extended Lagrangian defined by $\hat{L}(q, \dot{q})=L(q, \dot{q})-\lambda^{\top} \Phi(q, \dot{q})$ with a Lagrange multiplier $\lambda \in \mathbf{R}^{m}$. Calculating an increment of an extended functional $\hat{F}$ concerning with $\hat{L}$ for a variation $X$, we have

$$
\delta \hat{F}(q)(X)=\int_{t_{0}}^{t_{1}}\{\hat{L}(q+X, \dot{q}+\dot{X})-\hat{L}(q, \dot{q})\} d t=\int_{t_{0}}^{t_{1}}\left\langle\frac{\partial \hat{L}}{\partial q}-\frac{d}{d t}\left(\frac{\partial \hat{L}}{\partial \dot{q}}\right), X\right\rangle d t .
$$

From the Lagrange multiplier theorem, we can see that a curve $\tilde{q}^{*} \in \tilde{C}\left(q_{0}, q_{1}, I, P\right)$ is a solution of the vakonomic constrained variational problem if and only if $\delta \hat{F}(q)(X)=0$ holds. Since $\delta \hat{F}(q)(X)=0$ must be satisfied for all variation $X$, we obtain (14).

Substituting the extended Lagrangian (15) into the vakonomic equation (14), we obtain another representation of the vakonomic equations as follows:

$$
\frac{d}{d t}\left(\frac{\partial L}{\partial \dot{q}}\right)-\frac{\partial L}{\partial q}-\frac{d}{d t} \frac{\partial}{\partial \dot{q}}\left\{\lambda^{\top} \Phi(q, \dot{q})\right\}+\frac{\partial}{\partial q}\left\{\lambda^{\top} \Phi(q, \dot{q})\right\}=0 .
$$

Figure 1 [b] depicts an image on derivation of the equations of motion in vakonomic mechanics. It is noted that in vakonomic mechanics only variations that belong to the constrained space are considered from the beginning. It must be noted that in the vakonomic equation (16) the Lagrange multiplier $\lambda$ cannot be eliminated unlike the nonholonomic equation. So, we have to treat the Lagrange multiplier as an additional variable of the system.

Basically, nonholonomic and vakonomic mechanics yield different equations of motion. However, for the integrable constraints, the following result has been known.

Proposition 1 Assume that the constraints (6) are completely integrable. Then, the nonholonomic equation (10) is equivalent to the vakonomic equation (14) with the substitution $\mu=\dot{\lambda}$.

On the other hand, for nonintegrable constraints, it is known that the nonholonomic and vakonomic equations for the system of a rolling coin on a plane are equivalent to each other only when initial conditions of the Lagrange multipliers are set as 0 . However, for a free particle subject to a nonintegrable constraint in the 3-dimensional space called Heisenberg system, two kinds of equations 
of motion are not equivalent to each other for any choice of initial conditions of the Lagrange multipliers. In general, the physical difference between nonholonomic and vakonomic mechanics is less well understood. Theoretical analysis of vakonomic mechanics can be found in [10-18].

\section{Ball on rotating table}

\subsection{Problem setting}

In this section, we will derive two types of models for "a ball on a rotating table," which is one of the well-known nonholonomic systems, via both nonholonomic and vakonomic mechanics $[5,9,19]$. First, in this subsection, we give the problem setting for the system. As shown in Fig. 2, we deal with an undeformable ball and a rotating table that turns at a constant angular velocity. We assume that the ball does not slip and rotates with a velocity received by the rotating table. Consider the $x-y$ coordinate system so that the origin of it $O$ is coincident with the center of the rotating table, and let $(x, y)$ be the point with which the ball contacts. We denote the angles of rotation of the ball by $\left(\theta_{1}, \theta_{2}, \theta_{3}\right)$ as rotational angles about the $x, y$ and $z$ axes. This parameterization of rotation of the ball is based on "quasi-coordinates," and see $[1,4,5,9,19]$ for more details of it. Hence, the generalized coordinates of the system are represented by $q=\left[\begin{array}{lllll}x & y & \theta_{1} & \theta_{2} & \theta_{3}\end{array}\right]^{\top} \in \mathbf{R}^{2} \times \mathrm{SO}(3)$ with $n=5$.
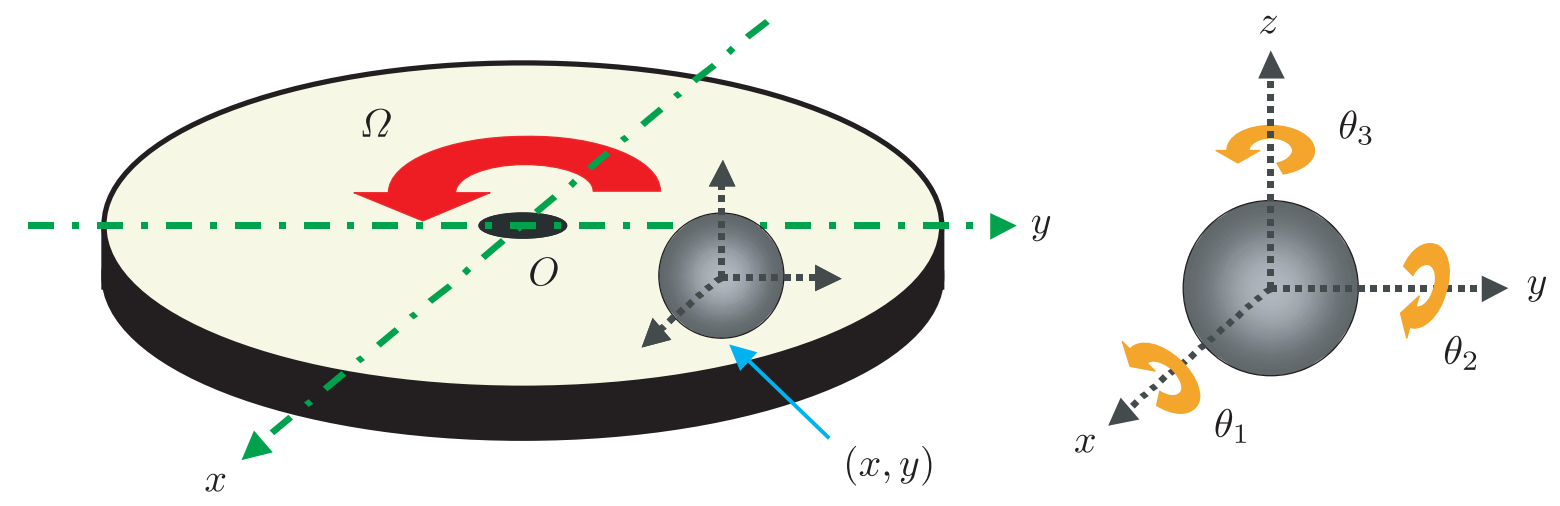

Fig. 2. A ball on a rotating table.

We use the parameters of the system; $R$ : the radius of the ball, $M$ : the mass of the ball, $I$ : the inertia moment of the ball, $\Omega$ : the angular velocity of the rotating table. The Lagrangian of the system is given by

$$
L(q, \dot{q})=\frac{1}{2} M\left(\dot{x}^{2}+\dot{y}^{2}\right)+\frac{1}{2} I\left(\dot{\theta}_{1}^{2}+\dot{\theta}_{2}^{2}+\dot{\theta}_{3}^{2}\right)
$$

as a sum of a kinetic energy and a rotational energy.

Next, we derive constraints to which the system is subjected. By considering the equilibrium of the velocities for the ball as shown in Fig. 3, we can obtain the constraints:

$$
\left\{\begin{array}{l}
\dot{x}-r \dot{\theta}_{2}=-\Omega y, \\
\dot{y}+r \dot{\theta}_{1}=\Omega x,
\end{array},\right.
$$

and thus $m=2$. We can see that (18) are represented by the form of affine constraints $A(q)+B(q) \dot{q}=$ 0 [20-26]. For the constraints (18), we can derive the following proposition.

Proposition 2: The affine constraints for a ball on a rotating table (18) are completely nonintegrable, that is, (18) are nonholonomic constraints.

(Proof) Consider a general class of affine constraints:

$$
A(q)+B(q) \dot{q}=0,
$$



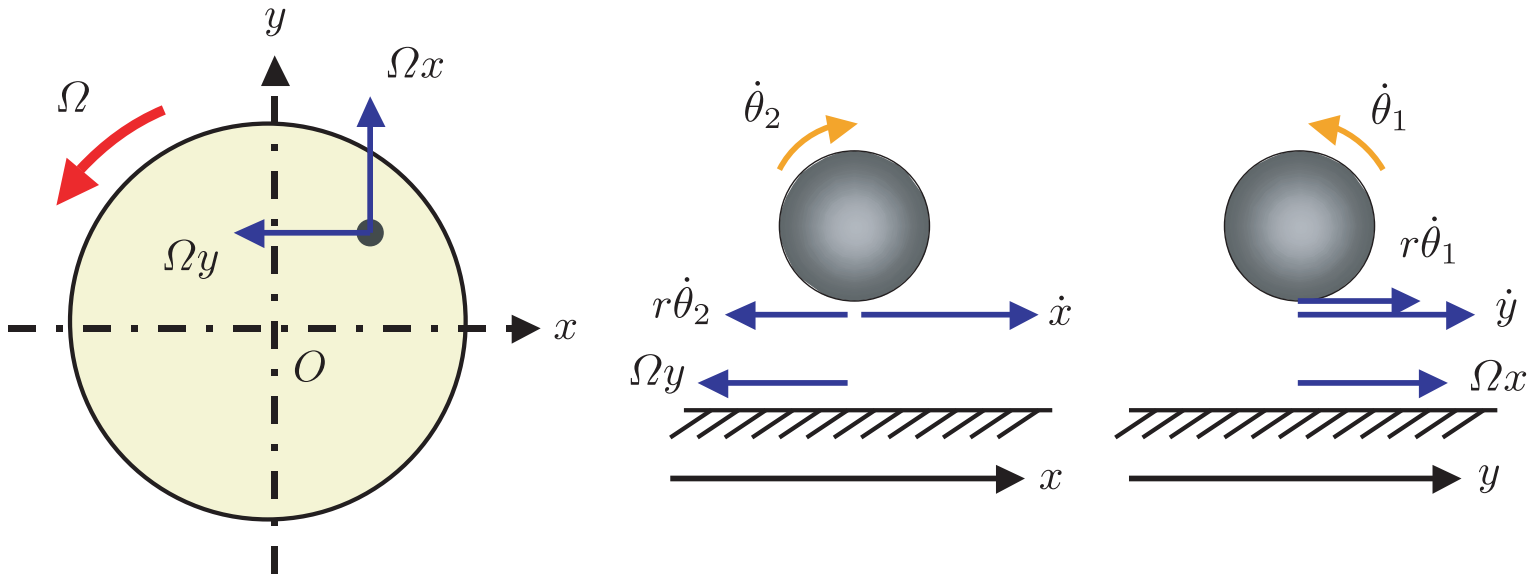

Fig. 3. Constraints for a ball on a rotating table.

where $q \in \mathbf{R}^{n}, A(q) \in \mathbf{R}^{m}, B(q) \in \mathbf{R}^{m \times n}$. Under the assumption that the $n-m$ row vectors of $B(q)$ in the affine constraints (19) are independent of each other at any point $q \in Q$, we can consider $m$ vectors which are independent of each other and annihilators of the $n-m$ row vectors of $B(q)$, and denote them by $Y_{1}, \cdots, Y_{m}$ as vector fields on $Q$. We now define a distribution $D(q)$ as $D=\operatorname{span}\left\{Y_{1}, \cdots, Y_{n-m}\right\}$, and a vector field $X$ that satisfies $A(q)+B(q) X(q)=0$ for its components. It is known that the affine constraints (19) can be geometrically represented by a pair $(D, X)$. Moreover, we define a smallest and involutive distribution $C_{0}(q)$ which contains $Y_{1}, \cdots, Y_{n-m}$ and satisfies $[X, W] \in C_{0}, \forall W \in C_{0}$, that is, $C_{0}$ is spanned by all the Lie brackets of $X, Y_{1}, \cdots, Y_{n-m}$ with the exception of $X$. Then, as shown in $[21,22]$, a necessary and sufficient condition of complete nonholonomicity for the affine constraints (19) is given by

$$
\operatorname{dim} C_{0}(q)=n .
$$

For the system of a ball on a rotating table, from (18) $A(q)$ and $B(q)$ are given by

$$
A(q)=\left[\begin{array}{c}
\Omega y \\
-\Omega x
\end{array}\right], B(q)=\left[\begin{array}{ccccc}
1 & 0 & 0 & -r & 0 \\
0 & 1 & r & 0 & 0
\end{array}\right],
$$

and hence vector fields $X, Y_{1}, Y_{2}, Y_{3}$ can be calculated as

$$
X=\left[\begin{array}{c}
-\Omega y \\
\Omega x \\
0 \\
0 \\
0
\end{array}\right], Y_{1}=\left[\begin{array}{l}
r \\
0 \\
0 \\
1 \\
0
\end{array}\right], Y_{2}=\left[\begin{array}{c}
0 \\
r \\
-1 \\
0 \\
1
\end{array}\right], Y_{3}=\left[\begin{array}{l}
0 \\
0 \\
0 \\
0 \\
1
\end{array}\right]
$$

Lie brackets of these vector fields are given by

$$
\left[X, Y_{1}\right]=\left[\begin{array}{c}
0 \\
-\Omega r \\
0 \\
0 \\
0
\end{array}\right],\left[X, Y_{2}\right]=\left[\begin{array}{c}
\Omega r \\
0 \\
0 \\
0 \\
0
\end{array}\right]
$$

Therefore, by setting

$$
C_{0}=\operatorname{span}\left\{Y_{1}, Y_{2}, Y_{3},\left[X, Y_{1}\right],\left[X, Y_{2}\right]\right\}
$$

we can see that $\operatorname{dim} C_{0}=5=n$ holds, and hence the affine constraints (18) are completely nonintegrable.

We now introduce two kinds of friction in order to obtain better models of the system. In [9], the authors used three types of friction: translational viscous friction, rolling friction, and rotational viscous friction. In this paper, we focus on translational viscous friction and rotational viscous friction 
that particularly influence behaviors of the system. Translational viscous friction occurs between the ball in translational motion and the table and is modeled by

$$
\left\{\begin{array}{l}
F_{1}=-\nu \dot{x} \\
F_{2}=-\nu \dot{y}
\end{array}\right.
$$

where $\nu$ is a coefficient of translational viscous friction. On the other hand, rotational viscous friction models air resistance that occurs between the ball in rotational motion and the table. The friction is modeled by

$$
\left\{\begin{array}{l}
T_{1}=-\xi \dot{\theta}_{1}, \\
T_{2}=-\xi \dot{\theta}_{2},
\end{array}\right.
$$

where $\xi$ is a coefficient of rotational viscous friction.

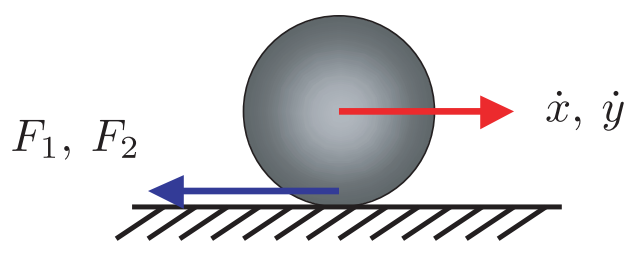

[a] Translational Viscous Friction

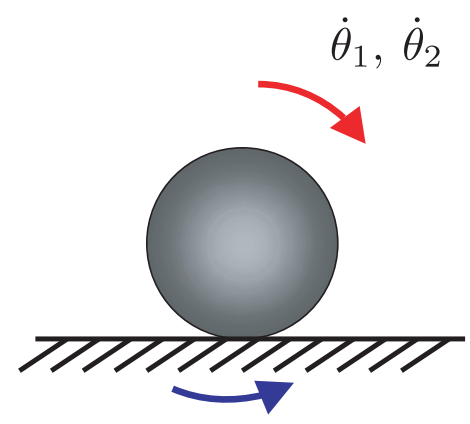

$T_{1}, T_{2}$

[b] Rotational Viscous Friction

Fig. 4. Translational and rotational viscous frictions.

\subsection{Modeling via nonholonomic and vakonomic mechanics}

In this subsection, we derive two models of the system of a ball on a rotating table by using nonholonomic and vakonomic mechanics based on the problem setting explained in the previous subsection. These two equations of motion are derived in [9]. In order to derive the equations of motion, we set the constraints (18) as

$$
\Phi(q, \dot{q})=\left[\begin{array}{c}
\Omega y+\dot{x}-r \dot{\theta}_{2} \\
-\Omega x+\dot{y}+r \dot{\theta}_{1}
\end{array}\right] .
$$

First, we derive a model of the system via nonholonomic mechanics. Substituting the Lagrangian (17) into the nonholonomic equation (10) and adding the translational and rotational viscous frictions (25), (26) to the right-hand side, we have

$$
\left\{\begin{array}{l}
M \ddot{x}=\mu_{1}-\nu \dot{x}, \\
M \ddot{y}=\mu_{2}-\nu \dot{y}, \\
I \ddot{\theta}_{1}=r \mu_{2}-\xi \dot{\theta}_{1}, \\
I \ddot{\theta}_{2}=-r \mu_{1}-\xi \dot{\theta}_{2}, \\
I \ddot{\theta}_{3}=0,
\end{array}\right.
$$

where $\mu_{1}, \mu_{2}$ are the Lagrange multipliers . In addition, eliminating the variables $\theta_{1}, \theta_{2}, \theta_{3}, \mu_{1}, \mu_{2}$ and the derivatives of them from (28) using the nonholonomic constraints (18), then we obtain the reduced nonholonomic model for $x, y$ and their derivatives:

$$
\left\{\begin{array}{l}
\left(I+M r^{2}\right) \ddot{x}+\left(\xi+\nu r^{2}\right) \dot{x}+I \Omega \dot{y}+\Omega \xi y=0 \\
\left(I+M r^{2}\right) \ddot{y}-I \Omega \dot{x}+\left(\xi+\nu r^{2}\right) \dot{y}-\Omega \xi x=0 .
\end{array}\right.
$$


It is noted that in the nonholonomic model (29), the Lagrange multipliers $\mu_{1}, \mu_{2}$ are eventually eliminated.

Next, we derive another model of the system via vakonomic mechanics. Substituting the Lagrangian (17) into the vakonomic equation (16) and adding the translational and rotational viscous frictions (25), (26) to the right-hand side, we get

$$
\left\{\begin{array}{l}
M \ddot{x}-\dot{\lambda}_{1}-\Omega \lambda_{2}=-\nu \dot{x}, \\
M \ddot{y}-\dot{\lambda}_{2}+\Omega \lambda_{1}=-\nu \dot{y}, \\
I \ddot{\theta}_{1}-r \dot{\lambda}_{2}=-\xi \dot{\theta}_{1}, \\
I \ddot{\theta}_{2}+r \dot{\lambda}_{1}=-\xi \dot{\theta}_{2}, \\
I \ddot{\theta}_{3}=0,
\end{array}\right.
$$

where $\lambda_{1}, \lambda_{2}$ are the Lagrange multipliers. Contrary to nonholonomic mechanics, it is uncertain how to add external forces such as frictions in vakonomic mechanics. So we have added different frictions to the vakonomic model just like the nonholonomic mechanics case. Then, eliminating the variables $\theta_{1}, \theta_{2}, \theta_{3}, \mu_{1}, \mu_{2}$ and their derivatives from (30) by the nonholonomic constraints (18), we obtain the reduced vakonomic model for $x, y, \lambda_{1}, \lambda_{2}$ and the derivatives of them:

$$
\left\{\begin{array}{l}
\left(I+M r^{2}\right) \ddot{x}+\left(\xi+\nu r^{2}\right) \dot{x}+I \Omega \dot{y}+\Omega \xi y-\Omega r^{2} \lambda_{2}=0, \\
\left(I+M r^{2}\right) \ddot{y}-I \Omega \dot{x}+\left(\xi+\nu r^{2}\right) \dot{y}-\Omega \xi x+\Omega r^{2} \lambda_{1}=0, \\
\left(I+M r^{2}\right) \dot{\lambda}_{1}+I \Omega \lambda_{2}-(I \nu-M \xi) \dot{x}+I M \Omega \dot{y}+M \Omega \xi y=0, \\
\left(I+M r^{2}\right) \dot{\lambda}_{2}-I \Omega \lambda_{1}-I M \Omega \dot{x}+(I \nu-M \xi) \dot{y}-M \Omega \xi x=0 .
\end{array}\right.
$$

It must be noted that unlike the nonholonomic model (29), the Lagrange multipliers $\lambda_{1}, \lambda_{2}$ remain in the vakonomic model (31), hence we need initial values of $\lambda_{1}, \lambda_{2}$ in simulations of (31).

\section{Simulations and theoretical analysis}

\subsection{Setting for simulations}

In this section, we will perform some simulations for the nonholonomic and vakonomic models of a ball on a rotating table, which are derived in the previous section, and we compare between two mechanics from simulation results. Moreover, we give theoretical analysis on behaviors of the two models.

First, this subsection explains the problem setting for simulations. For the ball, we use two types of materials: aluminum and steel, and two types of radii: $1.0 \mathrm{~cm}$ and $1.5 \mathrm{~cm}$, that is, four different balls are utilized in simulations as shown in the following table. In experiments in the next section, we use the same balls.

Table I. Balls in Simulations and Experiments.

\begin{tabular}{|c|c|c|c|c|}
\hline & Material & Radius $[\mathrm{cm}]$ & Density $\left[\mathrm{g} / \mathrm{cm}^{3}\right]$ & Mass $[\mathrm{g}]$ \\
\hline$[1]$ & Aluminum & 1.0 & 2.7 & 11.3 \\
\hline$[2]$ & Aluminum & 1.5 & 2.7 & 38.2 \\
\hline$[3]$ & Steel & 1.0 & 7.8 & 32.7 \\
\hline$[4]$ & Steel & 1.5 & 7.8 & 110.3 \\
\hline
\end{tabular}

The radius of the rotating table is $20 \mathrm{~cm}$ as shown in Fig. 5, and we use five kinds of the angular velocities of the rotating table: $\Omega=40,80,120,160,200 \mathrm{rpm}$. The common initial condition for the both models is $(x, y, \dot{x}, \dot{y})=(10,0,0,5)$, and for only the vakonomic model we use the nine types of the initial conditions of the Lagrange multipliers $\lambda_{1}, \lambda_{2}$ as

$$
\begin{aligned}
\left(\lambda_{1}, \lambda_{2}\right)= & (0,0),(0,10),(0,50),(10,0),(50,0), \\
& (10,10),(10,50),(50,10),(50,50) .
\end{aligned}
$$




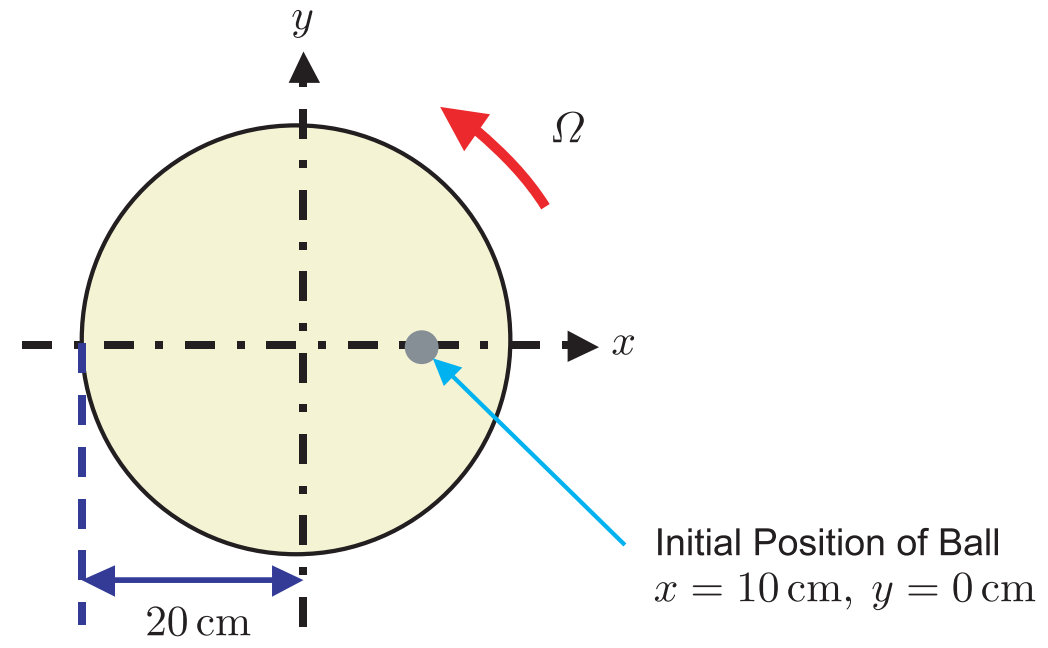

Fig. 5. Setting of simulations.

The simulation time is set as $30 \mathrm{~s}$, and simulations terminate when the balls reach the position of $23 \mathrm{~cm}$ from the center of the rotating table.

\subsection{Simulation results}

Simulations for the nonholonomic model (29) and the vakonomic model (31) are carried out based on the problem setting explained in the previous subsection. The implicit Runge-Kutta method is utilized for solving the equations of motion with the time step $h=0.01 \mathrm{~s}$. Simulation results are shown in Figs. 6 and 7 for the nonholonomic and vakonomic models, respectively. These figures show the solution trajectories of the balls in the $x-y$ plane. It is noted that in Fig. 7, nine solution trajectories for the initial values of the Lagrange multipliers are illustrated simultaneously.

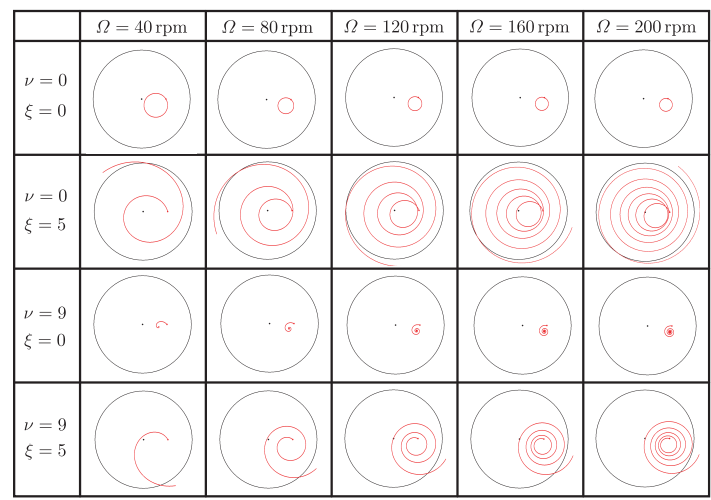

[a] Aluminium Ball : $r=1.0 \mathrm{~cm}$

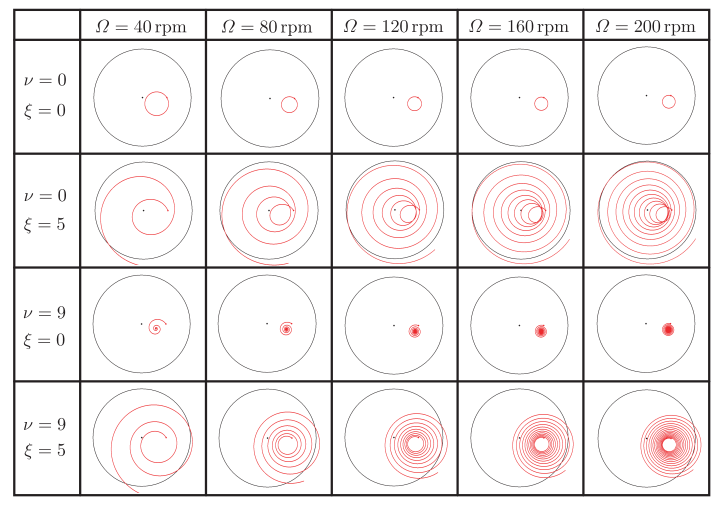

[c] Steel Ball : $r=1.0 \mathrm{~cm}$

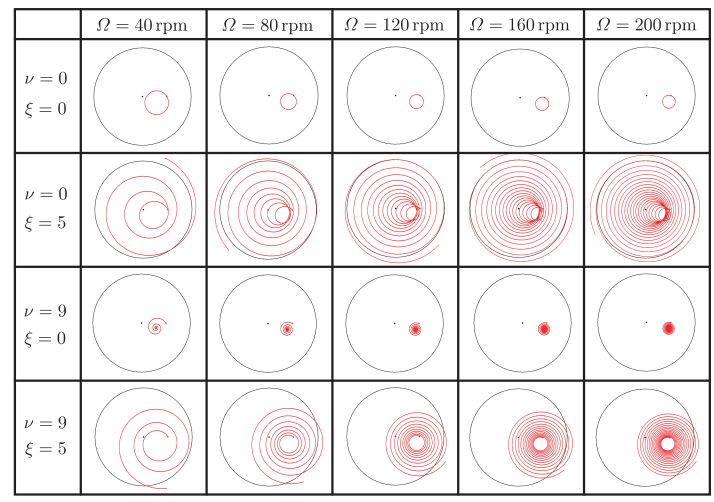

[b] Aluminium Ball : $r=1.5 \mathrm{~cm}$

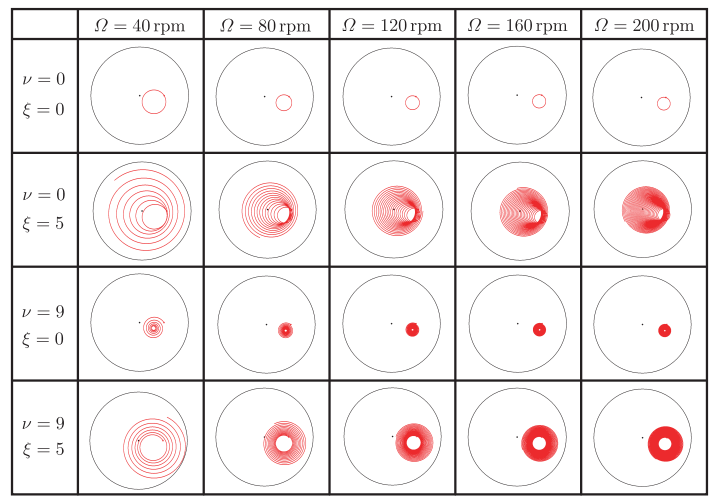

[b] Steel Ball : $r=1.5 \mathrm{~cm}$

Fig. 6. Simulation results for the nonholonomic model. 


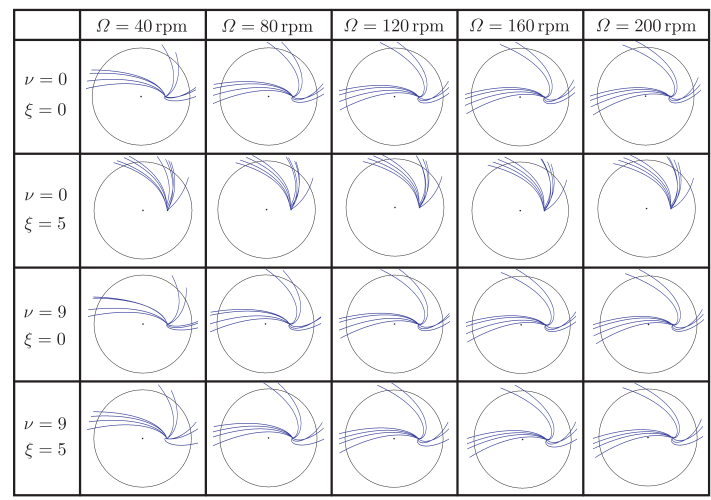

[a] Aluminium Ball : $r=1.0 \mathrm{~cm}$

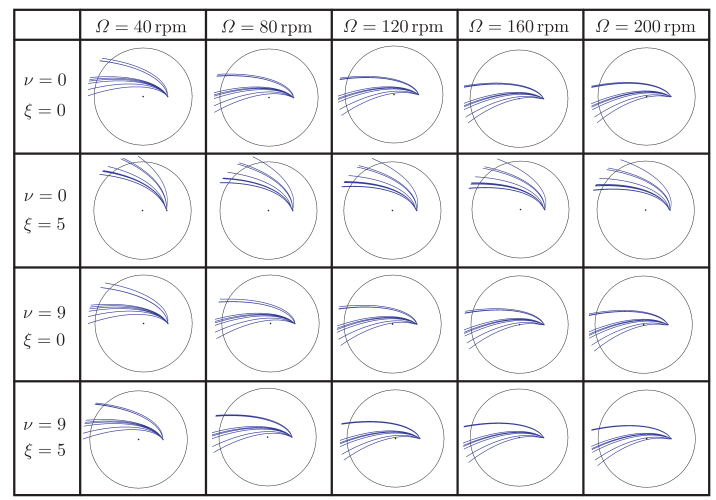

[c] Steel Ball : $r=1.0 \mathrm{~cm}$

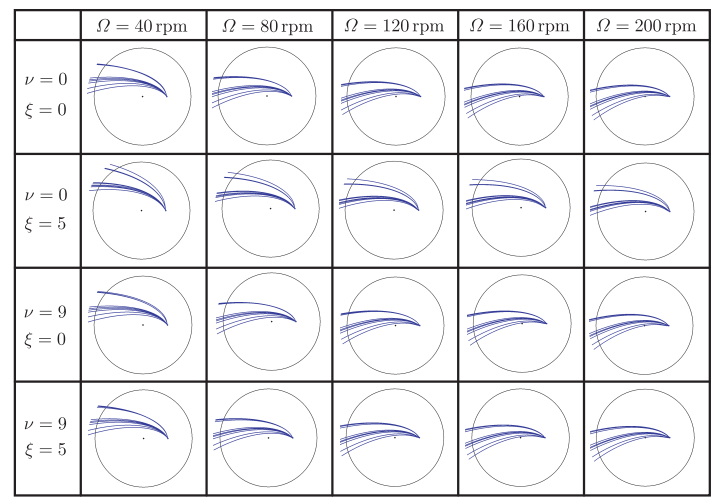

[b] Aluminium Ball : $r=1.5 \mathrm{~cm}$

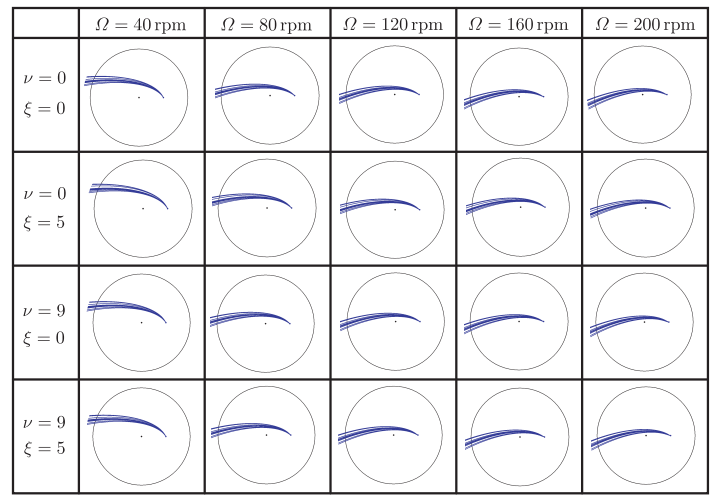

[b] Steel Ball : $r=1.5 \mathrm{~cm}$

Fig. 7. Simulation results for the vakonomic model.

From Fig. 6, for the nonholonomic model (29), we can find some characteristics as follows:

- As the mass of the ball and the angular velocity of the rotating table get larger, the ball becomes slower in motion.

- In the absence of friction $(\nu=0, \xi=0)$, the solution trajectories become limit cycles, and they do not depend on the material or the radius of the balls.

- The translational viscous friction destabilizes the behaviors of balls $(\nu=0, \xi=5)$.

- The rotational viscous friction stabilizes the behaviors of balls $(\nu=9, \xi=0)$.

On the other hand, from Fig. 7, we can find some characteristics of the vakonomic model (31) as follows:

- As the mass of the ball gets larger, the ball becomes faster in motion.

- All the solution trajectories are unstable, that is, the balls move continuously away from their initial position from the start.

- The solution trajectories are less affected by the two frictions.

From the results depicted in Figs. 6 and 7 and the characteristics mentioned above, we can see that the simulation results for the nonholonomic and vakonomic models are completely different from one another.

\subsection{Theoretical analysis}

This subsection presents theoretical analysis for the models of a ball on a rotating table without frictions, and investigates the case where the nonholonomic and vakonomic models are equivalent to each other. 
First, we consider the nonholonomic model (29). We define new variables $z_{1}=x, z_{2}=y, z_{3}=$ $\dot{x}, z_{4}=\dot{y}$ in (29), and set the parameters as

$$
I=\frac{2}{5} M r^{2}, \nu=0, \xi=0,
$$

where we consider a ball whose density is uniform. Then, from (29), we can obtain the following linear system:

$$
\left[\begin{array}{c}
\dot{z}_{1} \\
\dot{z}_{2} \\
\dot{z}_{3} \\
\dot{z}_{4}
\end{array}\right]=\underbrace{\left[\begin{array}{cccc}
0 & 0 & 1 & 0 \\
0 & 0 & 0 & 1 \\
0 & 0 & 0 & -\frac{2 \Omega}{7} \\
0 & 0 & \frac{2 \Omega}{7} & 0
\end{array}\right]}_{A}\left[\begin{array}{l}
z_{1} \\
z_{2} \\
z_{3} \\
z_{4}
\end{array}\right]
$$

and the eigenvalues of the matrix $A$ are given by

$$
\alpha_{1}=\alpha_{2}=0, \alpha_{3}=\frac{2}{7} \Omega i, \quad \alpha_{4}=-\frac{2}{7} \Omega i,
$$

where $i$ denotes the imaginary unit. Hence, we can solve (34) as

$$
z(t)=\sum_{k=1}^{4} a_{k} e^{\alpha_{k} t},
$$

where $a_{k} \in \mathbf{R}^{4}(k=1, \cdots, 4)$ are coefficients which are determined by initial conditions.

Next, we deal with the vakonomic model (31). We define new variables $w_{1}=x, w_{2}=y, w_{3}=$ $\dot{x}, w_{4}=\dot{y}, w_{5}=\lambda_{1}, w_{6}=\lambda_{2}$ in (31), and use the same parameters as (33). Then, from (31), we can obtain the following linear system:

$$
\left[\begin{array}{c}
\dot{w}_{1} \\
\dot{w}_{2} \\
\dot{w}_{3} \\
\dot{w}_{4} \\
\dot{w}_{5} \\
\dot{w}_{6}
\end{array}\right]=\underbrace{\left[\begin{array}{cccccc}
0 & 0 & 1 & 0 & 0 & 0 \\
0 & 0 & 0 & 1 & 0 & 0 \\
0 & 0 & 0 & -\frac{2 \Omega}{7} & 0 & \frac{5 \Omega}{7 M} \\
0 & 0 & \frac{2 \Omega}{7} & 0 & -\frac{5 \Omega}{7 M} & 0 \\
0 & 0 & 0 & -\frac{2 \Omega M}{7} & 0 & -\frac{2 \Omega}{7} \\
0 & 0 & \frac{2 \Omega M}{7} & 0 & \frac{2 \Omega}{7} & 0
\end{array}\right]}_{B}\left[\begin{array}{c}
w_{1} \\
w_{2} \\
w_{3} \\
w_{4} \\
w_{5} \\
w_{6}
\end{array}\right],
$$

and the eigenvalues of the matrix $B$ are given by

$$
\left\{\begin{array}{l}
\beta_{1}=\beta_{2}=0 \\
\beta_{3}=\left(-\frac{\sqrt{10}}{7}+\frac{2}{7} i\right) \Omega, \beta_{4}=\left(-\frac{\sqrt{10}}{7}-\frac{2}{7} i\right) \Omega, \\
\beta_{5}=\left(\frac{\sqrt{10}}{7}+\frac{2}{7} i\right) \Omega, \beta_{6}=\left(\frac{\sqrt{10}}{7}-\frac{2}{7} i\right) \Omega .
\end{array}\right.
$$

Hence, we can solve (37) as

$$
w(t)=\sum_{k=1}^{6} b_{k} e^{\beta_{k} t},
$$

where $b_{k} \in \mathbf{R}^{6}(k=1, \cdots, 6)$ are coefficients which are determined by initial conditions.

For the nonholonomic and vakonomic models (29), (31), we can derive the following proposition based on their linear systems (34), (37). 
Proposition 3 : For the system of a ball on a rotating table, a solution trajectory of the vakonomic model (31) without frictions is not unstable if and only if $\left(\dot{x}(0), \dot{y}(0), \lambda_{1}(0), \lambda_{2}(0)\right)=$ $(0,0,0,0)$ holds for initial conditions. Then, the solution is given by

$$
x(t)=x(0), y(t)=y(0) .
$$

Moreover, (40) is equivalent to a solution of the nonholonomic model (29) without frictions for the initial condition $(\dot{x}(0), \dot{y}(0))=(0,0)$.

(Proof) It turns out that unstability of solution trajectories of the vakonomic model (31) arises by the unstable eigenvalues $\beta_{5}, \beta_{6}$ in (38). So, we consider two equations which are derive by setting the first and second component of $b_{5}$ and $b_{6}$ as 0 . From these equations, we can obtain the next:

$$
\lambda_{1}(0)=-\frac{\sqrt{10}}{5} M i \cdot \dot{x}(0), \lambda_{2}(0)=-\frac{\sqrt{10}}{5} M i \cdot \dot{y}(0) .
$$

Since the values of $x, y, \lambda_{1}, \lambda_{2}$ should be real, then, from (41) the values of $\dot{x}(0), \dot{y}(0)$ should be also real. Hence, we have $\dot{x}(0)=\dot{y}(0)=0$ and $\lambda_{1}(0)=\lambda_{2}(0)=0$ from (41). Using these initial conditions, we obtain the solution (40) from (39). In addition, solving (34) with the initial condition $\dot{x}(0)=\dot{y}(0)=0$, we also have the same solution (40). Therefore, this proposition is proven.

From Proposition 3, we can see that if we choose initial values of the Lagrange multipliers $\lambda_{1}, \lambda_{2}$ so that a solution trajectory of the vakonomic model (31) is stable, only $\lambda_{1}(0)=\lambda_{2}(0)=0$ should be set and the solution trajectory (40) is trivial. Moreover, it is confirmed that only in this particular case a solution of the nonholonomic models (29) is equivalent to that of the vakonomic model (31), and hence behaviors of these two models are fundamentally different from one another. Similar analysis on the nonholonomic and vakonomic models was shown in [9], and the result gives close agreement with the one derived in this subsection.

\section{Experimental comparison}

\subsection{Setting for experiments}

In this section, we perform some experiments of a ball on a rotating table using a laboratory equipment, and compare between the simulation results in the previous section and the experimental results.

We first explain the setting of experiments in this subsection. In order to compare experimental results to the simulation ones, we fundamentally use the same parameters of the system. The laboratory equipment of the rotating table is illustrated in Fig. 8 [a] and the radius of the table is $20 \mathrm{~cm}$.

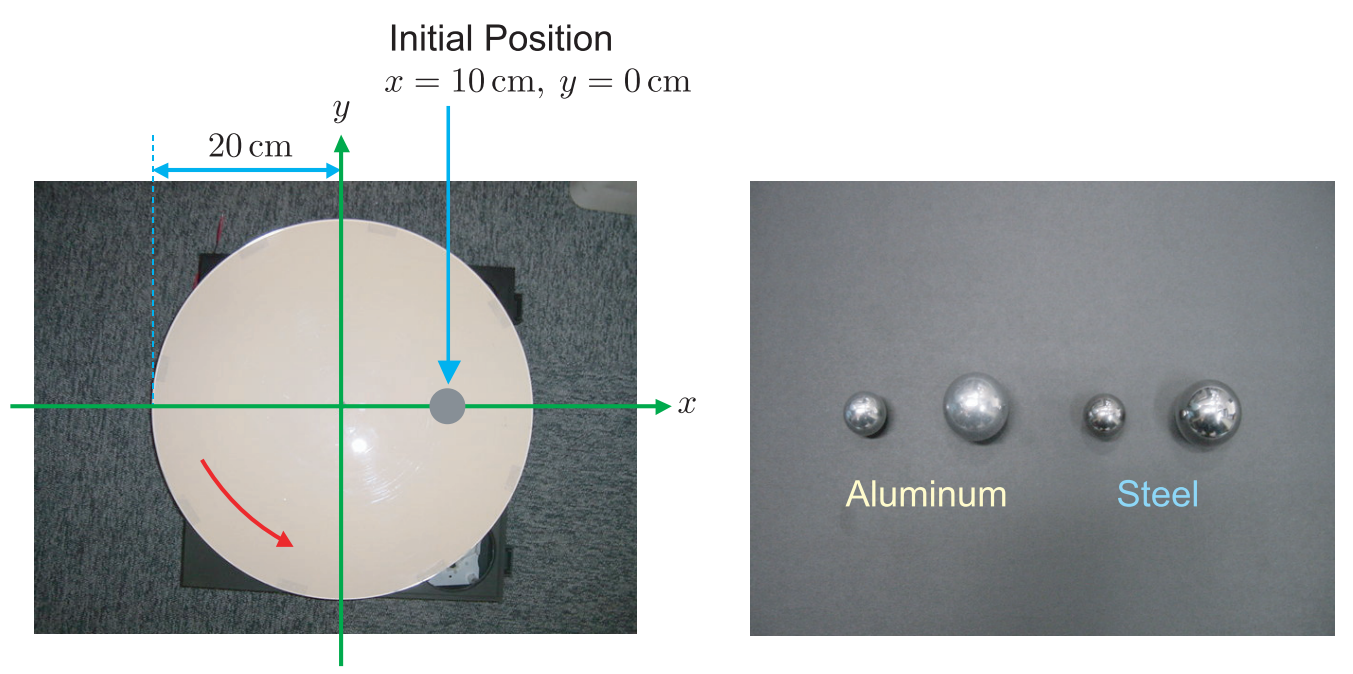

Fig. 8. Setting of the labratory equipment. 
The balls are depicted in Fig. 8 [b] and we use two types of materials: aluminum and steel, and two types of radii: $1.0 \mathrm{~cm}$ and $1.5 \mathrm{~cm}$ (see also Table I).

For the angular velocities of the rotating table, we use five values: $\Omega=40,80,120,160,200 \mathrm{rpm}$, that is to say, we carry out twenty trials of experiments. The initial position of the ball is set as $x=10 \mathrm{~cm}, y=0 \mathrm{~cm}$ as shown in Fig. 8 [a], and the initial velocity of the ball $\dot{x}=0 \mathrm{~cm} / \mathrm{s}, y=5 \mathrm{~cm} / \mathrm{s}$ is realized using an artificial perturbation. In addition, we do not consider the limitation of the simulation time $30 \mathrm{~s}$, which is used in the simulations in Section 4.

\subsection{Experimental results and discussion}

Some experiments are performed based on the problem setting described in the previous subsection using the laboratory equipment. The experimental results shown in Fig. 9 illustrate the trajectories of the ball. Moreover, a snapshot of the trajectory of the 1.0-cm steel ball $(\Omega=120 \mathrm{rpm})$ is depicted in Fig. 10. From these results, we can find some features as follows:

- The balls rotate in the counterclockwise direction with increasing radius of rotation from their initial point.

- As the mass of the ball and the angular velocity of the rotating table get larger, the ball becomes slower in motion.

- The balls themselves spin at high angular velocities.

Comparing the simulation results obtained in the previous section with the experimental results, we can see the following characteristics:

- The behaviors of the balls in the experimental results shown in Fig. 9 are similar to ones in the simulation results for the nonholonomic model with frictions (29), which are illustrated in the fourth row of each block in Fig. 6 .

- There are no trajectories that immediately move off the rotating table like the simulation results for the vakonomic model (31) as shown in Fig. 7.

Consequently, from the above discussions, we conclude that for the system of a ball on a rotating table, behaviors of the nonholonomic model (29) agree rather well with experimental results, and hence

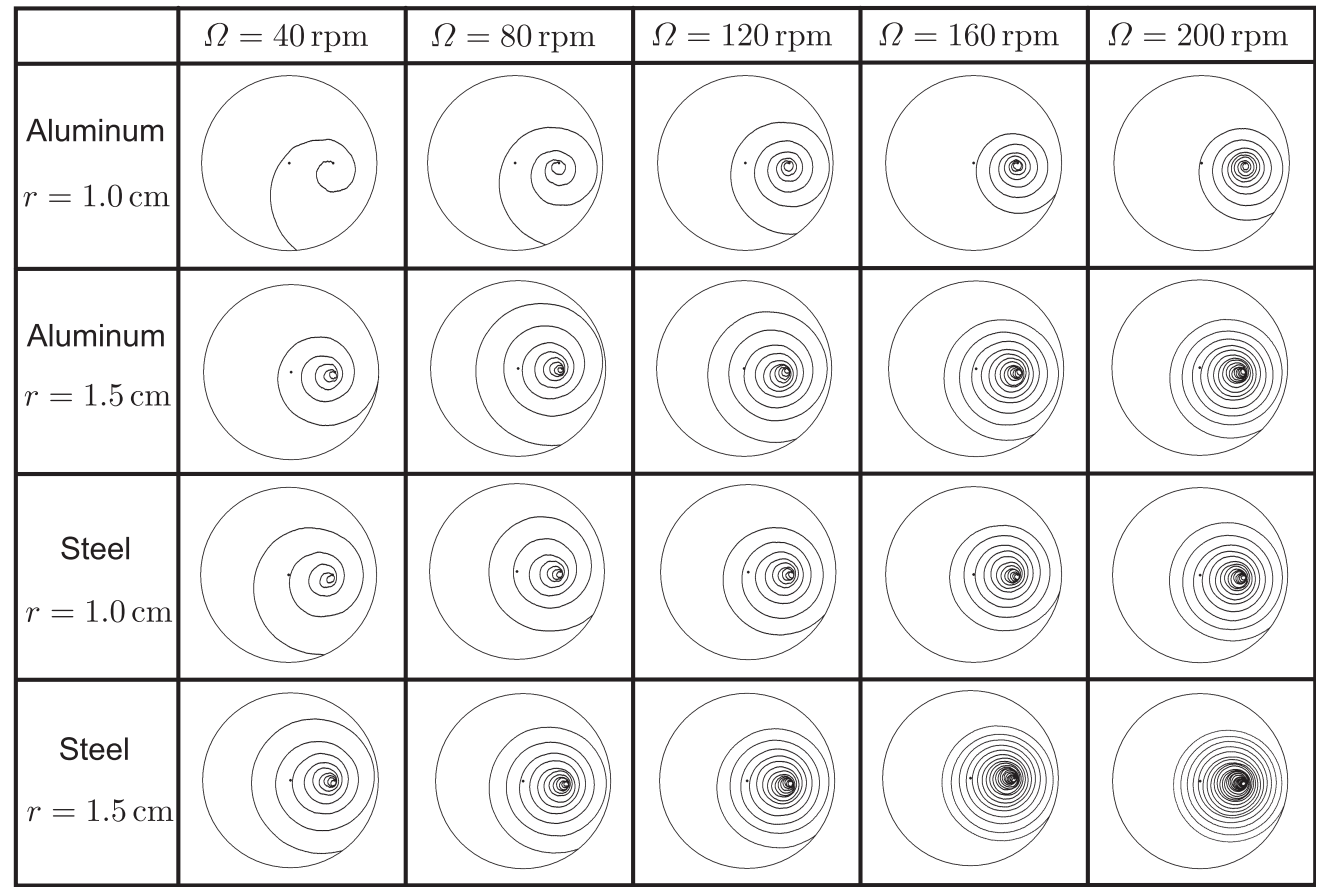

Fig. 9. Experimental results. 


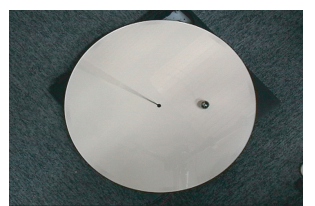

[1] $\mathrm{t}=0.0$

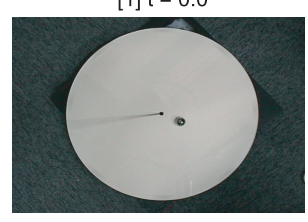

[6] $\mathrm{t}=2.5$

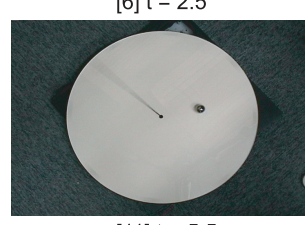

[11] $\mathrm{t}=5.5$

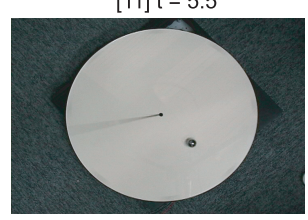

[16] $\mathrm{t}=8.0$

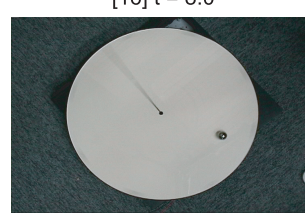

$[21] \mathrm{t}=10.5$

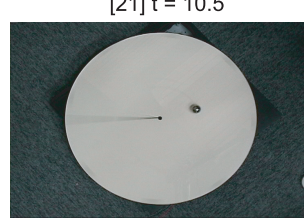

$[26] \mathrm{t}=13.0$

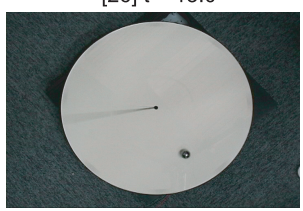

$[31] \mathrm{t}=15.5$

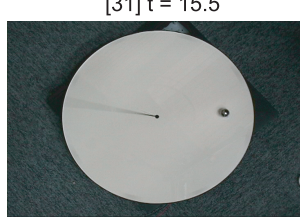

[36] $\mathrm{t}=18.0$

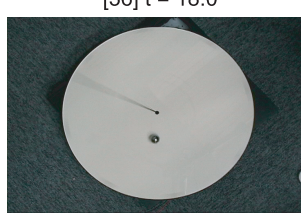

[41] $\mathrm{t}=20.5$

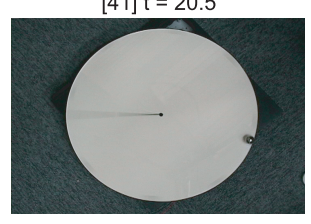

$[46] \mathrm{t}=23.0$

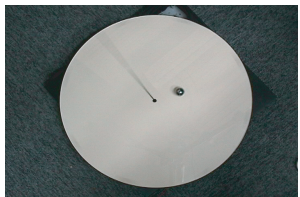

[2] $\mathrm{t}=0.5$

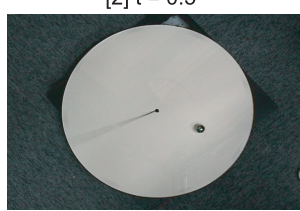

[7] $\mathrm{t}=3.0$

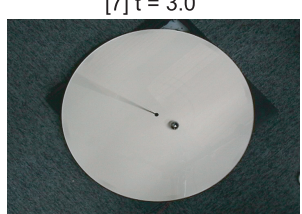

[12] $\mathrm{t}=6.0$

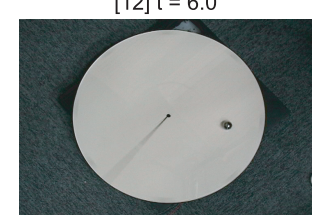

17] $t=8.5$

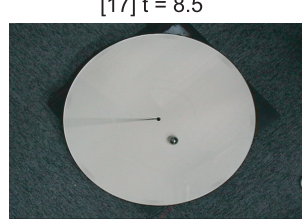

$[22] \mathrm{t}=11.0$

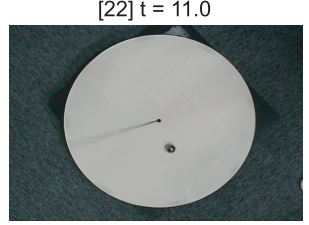

[27] $\mathrm{t}=13.5$

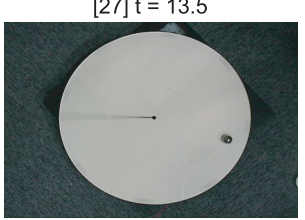

[32] $\mathrm{t}=16.0$

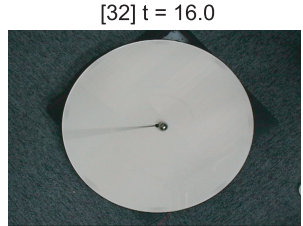

[37] $\mathrm{t}=18.5$

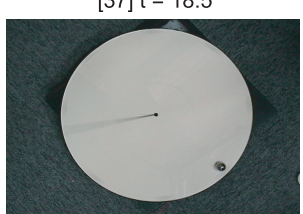

[42] $\mathrm{t}=21.0$

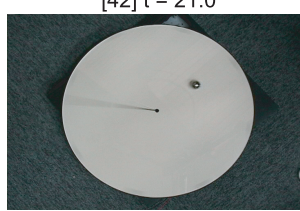

[47] $\mathrm{t}=23.5$

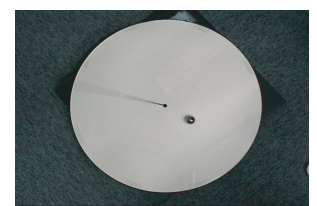

[3] $\mathrm{t}=1.0$

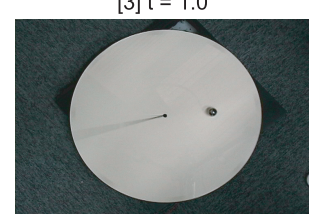

[8] $t=3.5$

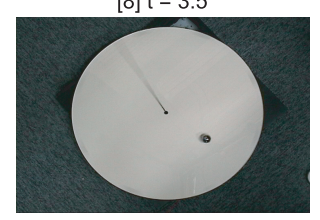

[13] $\mathrm{t}=6.5$
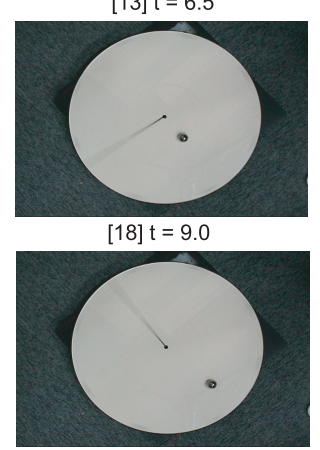

$[23] \mathrm{t}=11.5$

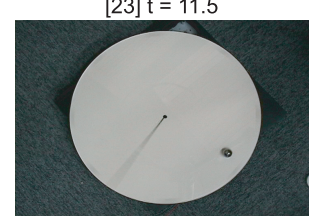

$[28] \mathrm{t}=14.0$

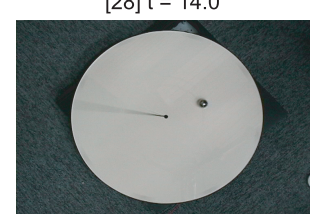

[33] $\mathrm{t}=16.5$

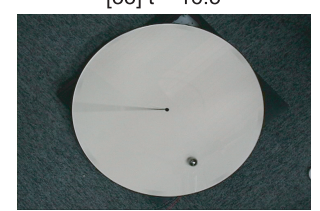

[38] $\mathrm{t}=19.0$

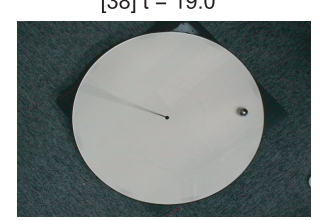

[43] $\mathrm{t}=21.5$

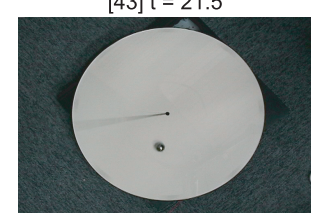

[48] $\mathrm{t}=24.0$

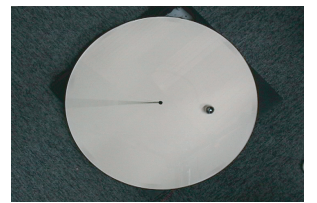

[4] $\mathrm{t}=1.5$

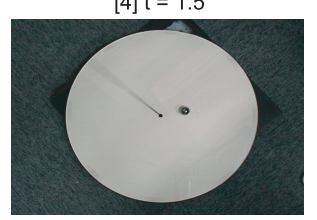

$[9] \mathrm{t}=4.0$
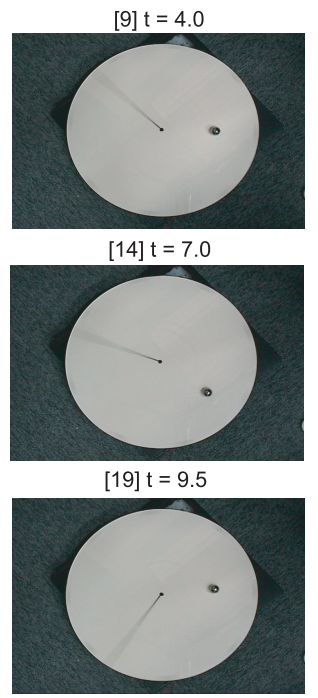

$[24] \mathrm{t}=12.0$

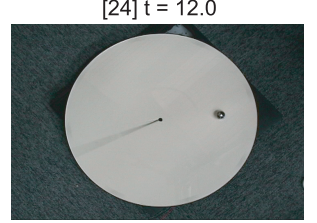

$[29] \mathrm{t}=14.5$

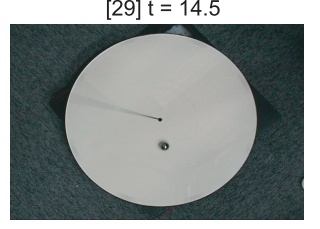

$[34] \mathrm{t}=17.0$

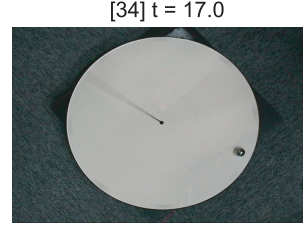

[39] $\mathrm{t}=19.5$
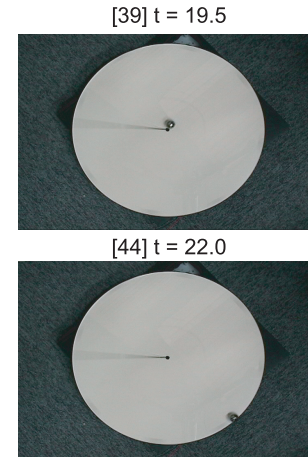

[49] $\mathrm{t}=24.5$

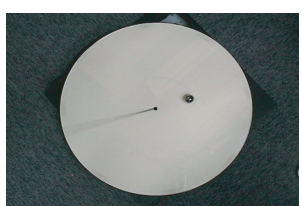

[5] $\mathrm{t}=2.0$

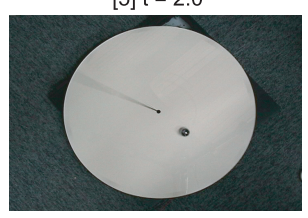

10] $t=4.5$

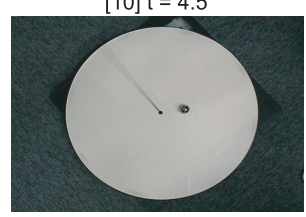

15] $t=7.5$

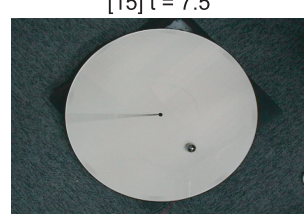

[20] $\mathrm{t}=10.0$

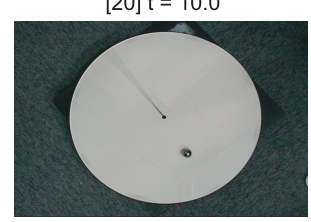

$[25] \mathrm{t}=12.5$

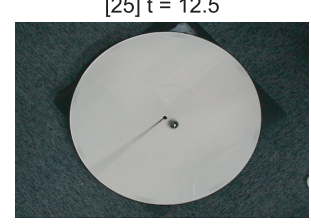

$[30] \mathrm{t}=15.0$

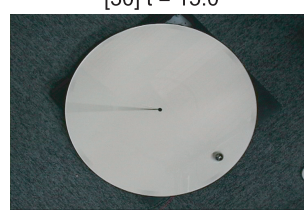

[35] $\mathrm{t}=17.5$

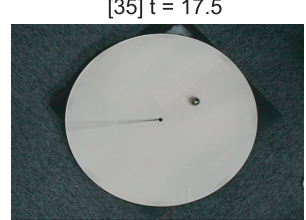

[40] $\mathrm{t}=20.0$

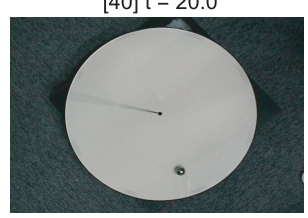

[45] $t=22.5$

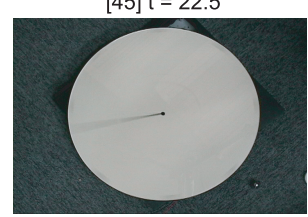

[50] $\mathrm{t}=25.0$

Fig. 10. Snapshots of a trajectory of the 1.0-cm steel ball. 
nonholonomic mechanics is a better modeling method than vakonomic mechanics for the system as Lewis and Murray's assertion [9].

\section{Conclusions}

In this paper, we have considered a comparison between nonholonomic and vakonomic mechanics via not only simulations but also experiments for the system of a ball on a rotating table. From these results, we conclude that nonholonomic mechanics provides a good model for the system, and confirmed Lewis and Murray's assertion [9]. However, that is not to say that vakonomic mechanics is totally wrong, and we would like to emphasize that vakonomic mechanics has possibilities and is strongly related to optimal control theory $[20,27,28]$ and sub-Riemannian geometry [29-31]. Hence, there still remain various problems on nonholonomic and vakonomic mechanics. Open problems and future work are as follows: experimental comparisons for other mechanical systems except a ball on a rotating table, theoretical analysis on the relationship between nonholonomic and vakonomic mechanics, investigations of vakonomic mechanics from the standpoints of optimal control theory and sub-Riemannian geometry.

\section{Acknowledgments}

This work was supported by JSPS KAKENHI Grant Number 24760339.

\section{References}

[1] J.E. Marsden and T.S. Ratiu, Introduction to Mechanics and Symmetry, Springer-Verlag, New York-Heidelberg-Berlin, 1994.

[2] J. Cortés, Geometric, Control and Numerical Aspects of Nonholonomic Systems, SpringerVerlag, 2002.

[3] A.M. Bloch, Nonholonomic Mechanics and Control, Springer-Verlag, 2003.

[4] F. Bullo and A.D. Rewis, Geometric Control of Mechanical Systems, Springer-Verlag, 2004.

[5] Ju. I. Neimark and N.A. Fafaev, Dynamics of Nonholonomic Systems, American Mathematical Society, 1972.

[6] V.I. Arnold, V.V. Kozlov, and A.I. Nejshtadt, Mathematical Aspects of Classical and Celestial Mechanics, Dynamical Systems III, Encyclopaedia of Mathematical Sciences, Springer Berlin, 1988.

[7] V.V. Kozlov, "Realization of nonintegrable constraints in classical mechanics," Sov. Phys. Dokl., no. 28, pp. 735-737, 1993.

[8] A.M. Bloch and P.E. Crouch, "Nonholonomic and vakonomic control systems on riemannian manifolds," Dynamics and Control of Mechanical Systems: The Falling Cat and Related Problems (Fields Institute Communications), pp. 25-52, 1993.

[9] A.D. Lewis and R.M. Murray, "Variational principles for constrained systems: theory and experiment," Int. J. Nonlinear Mechanics, vol. 30, no. 6, pp. 793-815, 1995.

[10] F. Cardin and M. Favretti, "On nonholonomic and vakonomic dynamics of mechanical systems with nonintegrable constraints," J. Geometry and Physics, vol. 18, no. 4, pp. 295-325, 1996.

[11] M. Leon, J. Marrero, and D. Diego, "Vakonomic mechanics versus non-holonomic mechanics: A unified geometrical approach," J. Geometry and Physics, vol. 35, no. 2-3, pp. 126-144, 2000.

[12] S. Martinez, J. Cortés, and M. Leon, "The geometrical theory of constraints applied to the vakonomic mechanical systems: The vakonomic bracket," J. Mathematical Physics, vol. 41, no. 4, pp. 2090-2120, 2000.

[13] G. Zampieri, "Nonholonomic versus vakonomic dynamics," J. Differential Equations, vol. 163, no. 2, pp. 335-347, 2000.

[14] I. Kupka and W. Oliva, "The non-holonomic mechanics," J. Differential Equations, vol. 169, no. 1, pp. 169-189, 2001.

[15] J. Cortes, M. Leon, D. Diego, and S. Martinez, "Geometric description of vakonomic and nonholonomic dynamics. comparison of solutions," SIAM J. Control and Optimization, vol. 41, no. 5, pp. 1389-1412, 2003. 
[16] Y.X. Guo, Y. Wang, G.Y. Chee, and F.X. Mei, "Nonholonomic versus vakonomic dynamics on a riemann-cartan manifold," J. Mathematical Physics, vol. 46, no. 6, 2005.

[17] R. Benito and D.M. de Diego, "Discrete vakonomic mechanics," J. Mathematical Physics, vol. 48, no. 8, 2005.

[18] S.M. Li and J. Berakdar, "On the validity of the vakonomic model and the chetaev model for constraint dynamical systems," Reports on Mathematical Physics, vol. 60, no. 1, pp. 107-116, 2007.

[19] V. Jurdjevic, Geometric Control Theory, Cambridge University Press, 1996.

[20] T. Kai and H. Kimura, "Constrained variational approach to nonholonomic control systems," in Proc. of the First International Conference "Physics and Control", St. Petersburg, Russia, pp. 1216-1221, 2003.

[21] T. Kai, H. Kimura, and S. Hara, "Nonlinear control analysis on kinematically asymmetrically affine control systems with nonholonomic affine constraints," in Proc. of 16th IFAC World Congress, Prague, Czech, Paper no. Mo-M08-TO/5, 2005.

[22] T. Kai, H. Kimura, and S. Hara, "Nonlinear control analysis on nonholonomic dynamic systems with affine constraints," in Proc. of 44th IEEE Conference on Decision and Control and European Control Conference 2005, Seville, Spain, pp. 1459-1464, 2005.

[23] T. Kai, "Affine constraints in nonlinear control theory," in Proc. of 3rd Workshop on Lagrangian and Hamiltonian Methods for Nonlinear Control, Nagoya, Japan, pp. 251-256, 2005.

[24] T. Kai, "Extended chained forms and their applications to nonholonomic kinematic systems with affine constraints," in Proc. of 45th IEEE Conference on Decision and Control, San Diego, USA, pp. 6104-6109, 2006.

[25] T. Kai, "Derivation and analysis of nonholonomic hamiltonian systems with affine constraints," in Proc. of European Control Conference 200\%, Kos, Greece, pp. 4805-4810, 2007.

[26] T. Kai, "Generalized canonical transformations and passivity-based control for nonholonomic hamiltonian systems with affine constraints," in Proc. of 46th IEEE Conference on Decision and Control, New Orleans, USA, pp. 3369-3374, 2007.

[27] D.E. Kirk, Optimal Control Theory: An Introduction, Dover Publications, 1970.

[28] L.D. Berkovitz, Optimal Control Theory, Springer US, 2010.

[29] A. Bellaiche and J.J. Risler, Sub-Riemannian Geometry, Birkhäuser, 1996.

[30] R. Montgomery, A Tour of Subriemannian Geometries, Their Geodesics and Applications, American Mathematical Society, 2006.

[31] O. Calin and D.C. Chang, Sub-Riemannian Geometry: General Theory and Examples, Cambridge University Press, 2009. 